\section{Humoral serological response to the BNT162b2 vaccine is abrogated in lymphoma patients within the first 12 months following treatment with anti-CD20 antibodies}

\author{
Ronit Gurion,, Uri Rozovski, ${ }^{1,2}$ Gilad Itchaki, ${ }^{1,2}$ Anat Gafter-Gvili, ${ }^{1,2,3}$ \\ Chiya Leibovitch, ${ }^{1,2}$ Pia Raanani,,2 Haim Ben-Zvi, ${ }^{4}$ Moran Szwarcwort, ${ }^{5}$ \\ Mor Taylor-Abigadol, ${ }^{6}$ Eldad J. Dann, ${ }^{6,7}$ Nurit Horesh, ${ }^{6}$ Tsofia Inbar, ${ }^{6}$ \\ Inna Tzoran, ${ }^{6,7}$ Noa Lavi, ${ }^{6,7}$ Riva Fineman, ${ }^{6}$ Shimrit Ringelstein-Harlev ${ }^{6,7 \#}$ \\ and Netanel A. Horowitz ${ }^{6,7 \#}$
}

${ }^{1}$ Institute of Hematology, Davidoff Center, Rabin Medical Center, Beilinson Hospital, Petach Tikva; ${ }^{2}$ Sackler School of Medicine, Tel Aviv University, Tel Aviv; ${ }^{3}$ Medicine A, Rabin Medical Center, Beilinson Hospital, Petach Tikva; ${ }^{4}$ Microbiology Laboratory, Rabin Medical Center, Beilinson Hospital, Petach Tikva; ${ }^{5}$ Virology Laboratory, Rambam Health Care Campus, Haifa; ' Department of Hematology and Bone Marrow Transplantation, Rambam Health Care Campus, Haifa and ${ }^{7}$ The Ruth and Bruce Rappaport Faculty of Medicine, Technion - Israel Institute of Technology, Haifa, Israel

${ }^{\text {"SR-H }}$ and NAH contributed equally as co-senior authors.
$\mathrm{P}$ atients with lymphoma, especially those treated with anti-CD20 monoclonal antibodies, suffer high COVID-19-associated morbidi- ty and mortality. The goal of this study was to assess the ability of lymphoma patients to generate a sufficient humoral response after two injections of BNT162b2 Pfizer vaccine and to identify factors influencing the response. Antibody titers were measured with the SARS-CoV-2 IgG II Quant $\left(\mathrm{Abbott}^{\circ}\right)$ assay in blood samples drawn from lymphoma patients $4 \pm 2$ weeks after the second dose of vaccine. The cutoff for a positive response was set at $50 \mathrm{AU} / \mathrm{mL}$. Positive serological responses were observed in $51 \%$ of the 162 patients enrolled in this cross-sectional study. In a multivariate analysis, an interval of $<12$ months between the last anti-CD20 monoclonal antibody dose and the second vaccine dose (odds ratio $=31.3$ [95\% confidence interval: $8.4-116.9], P<0.001$ ) and presence of active lymphoma (odds ratio $=4.2$ ( $95 \%$ confidence interval: 2.1 8.2), $P=0.006$ ) were identified as negative response predictors. The rate of seropositivity increased from $3 \%$ in patients vaccinated within 45 days after the last monoclonal antibody administration to $80 \%$ in patients vaccinated $>1$ year after this therapy. The latter percentage was equal to that of patients never exposed to monoclonal antibodies. In conclusion, lymphoma patients, especially those recently treated with antiCD20 monoclonal antibodies, fail to develop sufficient humoral response to BNT162b2 vaccine. While a serological response is not the only predictor of immunity, its low level could make this population more vulnerable to COVID-19, which implies the need for a different vaccination schedule for such patients.

\section{Introduction}

The global pandemic of coronavirus disease 2019 (COVID-19) had resulted in about 3.85 million deaths world-wide as of June 2021, with the estimated fatality rate among infected patients being between $1.5 \%$ and $2.1 \%{ }^{1}$ Emerging data demonstrate higher mortality rates among certain high-risk populations with significant co-morbidities, such as organ transplant recipients ${ }^{2}$ and cancer patients. ${ }^{3.5}$ There is evidence showing that patients with hematologic malignancies are the most vulnerable cancer population, ${ }^{3.7}$ with a higher risk of hospitalization and mortality following exposure to the virus. ' Estimated odds ratios (OR) for mortality are reported to vary between
Haematologica 2022

Volume 107(3):715-720

\section{Correspondence:}

NETANEL A. HOROWITZ

n_horowitz@rambam.health.gov.il

Received: May 12, 2021.

Accepted: June 22, 2021

Pre-published: July 29, 2021.

https://doi.org/10.3324/haematol.2021.279216

(C)2022 Ferrata Storti Foundation

Material published in Haematologica is covered by copyright. All rights are reserved to the Ferrata Storti Foundation. Use of published material is allowed under the following terms and conditions:

https://creativecommons.org/licenses/by-nc/4.0/legalcode. Copies of published material are allowed for personal or internal use. Sharing published material for non-commercial purposes is subject to the following conditions:

https://creativecommons. org//icenses/by-nc/4.0/legalcode, sect. 3. Reproducing and sharing published material for commercial purposes is not allowed without permission in writing from the publisher. 
2.09 and 12.16, depending on the type of malignancy and whether the disease has been actively treated within the months preceding the infection. ${ }^{5,6,8,9}$ Both non-Hodgkin lymphoma per se and prior chemotherapy with or without antiCD20 monoclonal antibodies have been suggested to contribute to patients' reduced survival and prolonged hospitalization following infection with severe acute respiratory syndrome coronavirus-2 (SARS-CoV-2). . $^{1013}$

The damage the pandemic inflicted on multiple healthcare systems which collapsed as a result of the high incidence of respiratory illness and intensive care demand, mostly due to the severity of COVID-19, led to an accelerated Food and Drug Administration approval of several anti-SARS-CoV-2 vaccines, following the successful completion of phase III studies. Among them was the BNT162b2 mRNA vaccine, which was demonstrated to have an efficacy of $95 \%$ in disease prevention in the pivotal phase III study. While the trial included approximately 40,000 volunteers, patients with active cancer were not enrolled into the study. ${ }^{14}$

Promptly after the Food and Drug Administration approval, this vaccine was approved by the Israeli Ministry of Health (December 2020), and vaccination was initiated at a large scale nation-wide level, with around $70 \%$ of the population aged 16 years and above having been fully vaccinated by April 2021. In addition, vaccination of potentially immunocompromised populations was started, including patients with hematologic conditions, despite the lack of good quality efficacy data for these patients, but in accordance with recommendations by hematologic and infectious disease agencies around the world..$^{15-17}$

The rationale for this action had been the emerging data regarding the high infection-related morbidity and mortality among these patients, especially during the periods of peak virus spread, along with the probable low risk of vaccine-induced complications. However, at the physiological level, it is unclear whether patients with lymphoma will be able to generate good quality immune responses to this vaccine, since the response to any vaccine requires interactions between various compartments of the immune system, many of which are compromised by the lymphoproliferative disease itself,, ${ }^{11}$ but even more so, by the chemoimmunotherapy regimens used for the treatment of these diseases. ${ }^{18,19}$ The lower prevalence and slower evolution of a humoral response to SARS-CoV-2 infection observed in this population of patient ${ }^{20,21}$ insinuate that this might be the case with humoral responses to the vaccine as well ${ }^{22}$.

The objectives of this study were to evaluate the rates of anti-spike (anti-S) antibody responses to the BNT162b2 vaccine among lymphoma patients and to identify patientand treatment-related factors influencing the antibody responses.

\section{Methods}

This was a non-interventional cross-sectional study conducted at two medical centers in Israel: Rambam Health Care Campus, Haifa (RMB) and Rabin Medical Center, Petach Tikva (RMC). All the procedures involved in this study were in accordance with the ethical standards of the institutional review boards of the two centers (approvals: \# 0883-20-RMB; 1087-20-RMC) and with the 1964 Helsinki Declaration and its later amendments. All patients signed the informed consent form.

The inclusion criteria were: age $\geq 18$ years, the diagnosis of a lymphoproliferative disease, including Hodgkin and non-Hodgkin lymphoma according to the World Health Organziation 2016 classification $^{23}$ and no known history of COVID-19 infection. Study participants were divided into the following two groups: (i) patients who received treatment, including chemotherapy or immunochemotherapy, i.e., monoclonal antibodies, tyrosine kinase inhibitors or immunomodulatory drugs, within 12 months prior to anti-COVID-19 vaccination; and (ii) patients with indolent lymphoma who were under "watch-and-wait" management before anti-COVID-19 vaccination.

All patients were vaccinated with two doses of BNT162 b2 vaccine, 21 days apart, and were followed at hematology clinics. Blood samples were drawn $4 \pm 2$ weeks after the second dose of vaccine and were evaluated for anti-spike SARS-CoV-2 antibodies. The SARS-CoV-2 IgG II Quant (Abbott ${ }^{\circ}$ ) assay was performed as per manufacturer's instructions for quantitative measurement of $\mathrm{IgG}$ antibodies against the spike protein of SARS-CoV-2. The test result was considered positive if the IgG level was $\geq 50 \mathrm{AU} / \mathrm{mL}$. The patients' baseline characteristics, collected from institutional electronic medical records, included each patient's demographics, comorbidities, lymphoma characteristics, duration, type and the first and last dates of anti-cancer treatment as well as disease activity before vaccination. Laboratory data such as complete blood count and serum protein electrophoresis before vaccination were also documented. The primary outcome was the rate of seropositivity for anti-spike antibodies.

\section{Statistical considerations}

We analyzed patients' characteristics using frequencies (percentages) for categorical variables and median (range) for continuous variables. A logistic regression model with the $\exp (\beta)$ was applied as an estimator of an OR and the $95 \%$ confidence interval ( $95 \% \mathrm{CI})$ around it to define the baseline variables that predict negativity of a serological response to SARS-CoV-2 vaccine. We used the likelihood ratio of the receiver operator characteristics curves and area under the curve to define the optimal cutoff for continuous variables. Univariate and multivariate logistic regression analyses were performed to evaluate potential predictors of seronegativity. To predict anti-spike IgG levels, we fitted a multiple-variable linear regression model based on: age, gender, lymphoma type, absolute lymphocyte count and time from the last anti-CD20 monoclonal antibody treatment to vaccination. Stepping method criteria for entry and removal were 0.05 and 0.2 , respectively. The Kruskal-Wallis test was used to compare medians of antibody titers. To generate $95 \%$ CI around proportions, we used the binomial approximation of the normal distribution. Statistical analyses were performed using SPSS software (version 27, SPSS inc. Chicago, IL, USA) and GraphPad Prism version 6.0 software (GraphPad Software, San Diego, CA, USA).

\section{Results}

\section{Patients' characteristics}

A total of 162 lymphoma patients who received two doses of the BNT162b2 vaccine between January and April, 2021 were included in the study. The median age of participants was 65 years (interquartile range, 52-73), 55\% were males, 142 (88\%) had non-Hodgkin lymphoma, including indolent and aggressive disease and the remaining 20 (12\%) had Hodgkin disease. Reported comorbidities included diabetes mellitus (19\%), ischemic heart disease $(11 \%)$, and other malignancies (17\%). Most (55\%) of the patients received first-line anti-lymphoma therapy, while about $17 \%$ were under "watch-and-wait" management. The most 
common treatment protocols included CHOP (cyclophosphamide, vincristine, adriamycin and prednisone) or bendamustine with or without anti-CD20 monoclonal antibodies, either rituximab or obinutuzumab. Few patients received other therapies, such as Bruton tyrosine kinase (BTK) inhibitors, lenalidomide or antiPD1 antibodies. The patients' characteristics are presented in Table 1.

\section{Serological response to vaccination}

Eighty-three patients (51\%) were seropositive (IgG levels $\geq 50 \mathrm{AU} / \mathrm{mL}$ ) and $49 \%$ had negative serology. In univariate

Table 1. Characteristics of the patients with lymphoma.

\begin{tabular}{lc} 
Characteristics & N (\%) \\
Age in years, median (IQR) & $65(52-73)$ \\
Males & $89(55 \%)$ \\
\hline Comorhidities & \\
Diabetes mellitus & $30(19 \%)$ \\
Ischemic heart disease & $17(11 \%)$ \\
\hline Hypertension & $54(34 \%)$ \\
Chronic renal failure & $12(7.5 \%)$ \\
\hline Chronic obstructive pulmonary disease & $7(4 \%)$ \\
Other malignancy & $27(17 \%)$ \\
\hline Type of lymphoma & \\
Diffuse large B-cell lymphoma & 32 \\
Follicular lymphoma & 64 \\
\hline Marginal zone lymphoma & 24 \\
Hodgkin lymphoma & 20 \\
\hline Peripheral T-cell lymphoma & 8 \\
Other lymphomas* & 14 \\
\hline
\end{tabular}

Line of treatment

\begin{tabular}{ll} 
Watch \& wait & 30 \\
First line & 89 \\
\hline Second line & 20 \\
Third line and beyond & 23 \\
\hline Type of treatment &
\end{tabular}

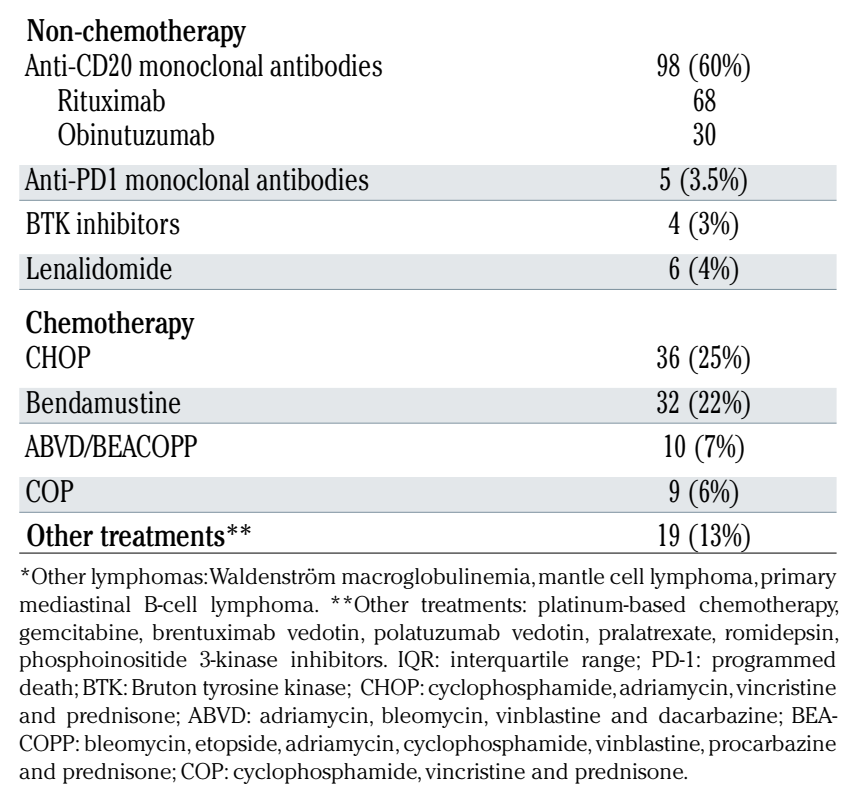

analysis, the following variables were found to be significantly associated with a lack of serological response: age $>80$ years $(\mathrm{OR}=4.3,95 \%$ CI: 1.1-1.6), absolute lymphocyte count $<1.2 \times 10^{9} / \mathrm{L}(\mathrm{OR}=2.3,95 \%$ CI: 1.1-4.4), IgG levels $<630 \mathrm{~g} / \mathrm{L}(\mathrm{OR}=15.8,95 \% \mathrm{CI}: 1.9-129.9)$, active disease (defined as being under treatment for remission induction or by a positive positron emission tomography/computed tomography result) at vaccination $(\mathrm{OR}=4.2,95 \% \mathrm{CI}$ : 2.1 8.2), a time period of $<12$ months between the last anti$\mathrm{CD} 20$ treatment and vaccination $(\mathrm{OR}=31.3,95 \% \mathrm{CI}$ : 8.4116.9), the use of obinutuzumab versus rituximab (OR $>4.54$ ), aggressive non-Hodgkin lymphoma versus Hodgkin lymphoma ( $\mathrm{OR}=15.4,95 \% \mathrm{CI}$ : 3.1-76.6) (Table 2). Lack of seroconversion was most frequent among patients suffering from aggressive lymphoma (63\%) followed by those with indolent lymphoma (54\%) and was lowest in patients with Hodgkin disease $(10 \%)$. With the negative response rate in the last group used as a reference, the $\mathrm{OR}$ of this variable for patients with indolent disease was 1.5 (not statistically significant), while it was as high as 15 for patients with aggressive lymphoma (statistically significant, $P<0.01$ ). The rates of negative serological responses in patients receiving $\mathrm{CHOP}$ relative to those treated with bendamustine, with or without anti-CD20 monoclonal antibodies, were $63 \%$ and $84 \%$, respectively $(P=0.056)$. In multivariate analysis, two variables remained statistically significant: a time period $<12$ months between the last anti-CD20 treatment and the second dose of vaccine, and presence of active lymphoma (Table 3).

\section{The effect of anti-CD20 treatment on vaccination results}

Among 98 patients who received anti-CD20 monoclonal antibodies, as the time period between the last dose of this treatment and vaccination became longer, the likelihood of seropositivity increased. The seropositivity rate was $80 \%$ in patients vaccinated at least 12 months after administration

Table 2. Univariate analysis of factors associated with a lack of serological response.

\begin{tabular}{|c|c|c|c|}
\hline Variable & $\begin{array}{l}\text { Reference } \\
\text { (95\% CI) }\end{array}$ & Odds ratio & P-value \\
\hline Age $\geq 80$ years & Age $<80$ years & $4.3(1.1-1.6)$ & 0.031 \\
\hline Gender: female & Male & $0.8(0.42-1.5)$ & 0.9 \\
\hline $\mathrm{ALC} \leq 1.2 \times 10^{9} / \mathrm{L}$ & $\mathrm{ALC}>1.2 \mathrm{G} / \mathrm{L}$ & $2.3(1.1-4.4)$ & 0.02 \\
\hline $\operatorname{IgG} \leq 630 \mathrm{~g} / \mathrm{L}$ & $\operatorname{IgG}>630 \mathrm{~g} / \mathrm{L}$ & $15.8(1.9-129.9)$ & 0.001 \\
\hline Active disease & Disease in remission & $4.2(2.1-8.2)$ & $<0.001$ \\
\hline $\begin{array}{l}\text { Time between the last } \\
\text { anti-CD20 treatment and } \\
\text { vaccination }<12 \text { months }\end{array}$ & $\begin{array}{l}>12 \text { months or } \\
\text { non-exposure to } \\
\text { anti-CD20 }\end{array}$ & $31.3(8.4-116.9)$ & $<0.001$ \\
\hline $\begin{array}{l}\text { Type of anti-CD20 MoAb: } \\
\text { obinutuzumab }\end{array}$ & Rituximab & $>4.54(\mathrm{NA})$ & 0.04 \\
\hline $\begin{array}{l}\text { Type of lymphoma - } \\
\text { indolent lymphoma* }\end{array}$ & Hodgkin lymphoma & $1.46(0.67-3.1)$ & 0.34 \\
\hline Aggressive lymphoma** & Hodgkin lymphoma & $15.4(3.1-76.6)$ & $<0.01$ \\
\hline $\begin{array}{l}\text { Time between the last } \\
\text { chemotherapy administration } \\
\text { and vaccination: }<19 \text { days }\end{array}$ & $>19$ days & $1.75(0.32-9.4)$ & 0.515 \\
\hline
\end{tabular}

*Indolent lymphoma included follicular lymphoma, marginal zone lymphoma, mantle cell lymphoma and Waldenström macroglobulinemia. ${ }^{* *}$ Aggressive lymphoma included diffuse large B-cell lymphoma, primary mediastinal large B-cell lymphoma and peripheral T-cell lymphoma. 95\% CI: $95 \%$ confidence interval; ALC: absolute lymphocyte count; NA: not applicable; MoAb: monoclonal antibody. 
of anti-CD20 monoclonal antibodies, while this rate was only $3 \%$ in patients vaccinated within 45 days after antiCD20 therapy (Table 4). It is noteworthy that the seropositivity rate in the former group was similar to that observed in lymphoma patients who had not received this treatment (i.e., were treated with chemotherapy only or were under "watch-and-wait" management).

None of the 28 patients treated with obinutuzumab developed a serological response in comparison to $62 \%$ seronegativity demonstrated in patients treated with rituximab within the same time frame.

\section{Levels of SARS-CoV-2 IgG}

In a linear regression model, a shorter time period between anti-CD20 therapy and vaccination predicted lower levels of anti-spike IgG and explained the $18 \%$ variance in antibody titers, while all other evaluable variables, such as age, gender, lymphoma type and absolute lymphocyte count had no predictive power. A correlation was revealed between the levels of circulating anti-spike IgG antibodies and the time between the last anti-CD20 treatment and vaccination. A significant difference was found between patients never exposed to anti-CD20 therapy (median of $1161 \mathrm{AU} / \mathrm{mL}$; range, $0-15,567$ ) or those receiving these agents more than 12 months prior to vaccination (median of $661 \mathrm{AU} / \mathrm{mL}$; range, $0-15,220$ ), relative to patients treated with these drugs within 12 months before vaccination: 0-45 days (median of $0 \mathrm{AU} / \mathrm{mL}$; range, $0-225$ ); 46-120 days (median of $0.7 \mathrm{AU} / \mathrm{mL}$; range, 0-1575); 121-180 days (median of $0.5 \mathrm{AU} / \mathrm{mL}$; range, 0-234); and 181-365 days (median of $0 \mathrm{AU} / \mathrm{mL}$; range, $0-373 \mathrm{AU} / \mathrm{mL}$ ) (Figure 1).

In a model taking into account age, gender, absolute lymphocyte count, disease activity and the time from the last anti-CD20 treatment to vaccination, only the last variable was statistically significant and predicted the titers of IgG antibodies.

\section{Discussion}

The current study, evaluating the antibody-mediated response in lymphoma patients who received two doses of BNT162b2 vaccine, showed that only $51 \%$ of these individuals developed seropositivity. These findings are in line with results of the studies assessing the efficacy of other anti-viral vaccines in the lymphoma setting. Indeed, studies assessing the efficacy of the influenza vaccine demonstrated insufficient humoral immunity and higher rates of overt

Table 4. Serological response compared between patients treated with anti-CD20 monoclonal antibodies and those who did not receive this treatment.

\begin{tabular}{|c|c|c|c|c|}
\hline $\begin{array}{l}\text { Time from anti- } \\
\text { CD20 therapy } \\
\text { to vaccination } \\
\text { (days) }\end{array}$ & $\begin{array}{c}\text { N. of } \\
\text { patients }\end{array}$ & $\begin{array}{l}\text { N. of } \\
\text { s patients } \\
\text { with positive } \\
\text { serology }\end{array}$ & $\begin{array}{l}\% \text { of patients } \\
\text { with positive } \\
\text { serology (CI) }\end{array}$ & $\begin{array}{l}\% \text { of patients } \\
\text { with negative } \\
\text { serology (CI) }\end{array}$ \\
\hline $0-45$ & 34 & 1 & $3(1-15)$ & $97(85-99)$ \\
\hline $46-120$ & 21 & 5 & $24(8-47)$ & $76(53-92)$ \\
\hline $121-180$ & 4 & 1 & $25(1-81)$ & $75(19-99)$ \\
\hline $181-365$ & 7 & 1 & $14(1-58)$ & $86(42-99)$ \\
\hline$>366$ & 21 & 17 & $81(58-95)$ & $19(5-42)$ \\
\hline No anti-CD20 therap & py 56 & 45 & $80(68-90)$ & $20(10-32)$ \\
\hline
\end{tabular}

Table 3. Multivariate analysis of factors associated with a lack of serological response.

\begin{tabular}{lcc} 
Variable & P-value & Oddis Ratio (95\% CI) \\
Age $\geq 80$ years & 0.5 & $2.8(0.13-61.9)$ \\
ALC $\leq 1.2 \times 10 \%$ & 0.4 & $2.1(0.4-10.4)$ \\
\hline Active disease & 0.006 & $11.8(2-67.6)$ \\
Time between the last anti-CD20 & $<0.001$ & $93(12.3-704.4)$ \\
treatment and vaccination & & \\
$<12$ months & &
\end{tabular}

Type of lymphoma 0.8 $1.2(0.25-6.1)$

95\% CI: $95 \%$ confidence interval; ALC: absolute lymphocyte count. The IgG variable was removed due to missing data

clinical disease in patients treated with chemotherapy, with only $10 \%$ of patients developing a sufficient antibody titer to at least one of the influenza A antigens, as compared to $45 \%$ in the control group. ${ }^{24}$ Moreover, lymphoma patients vaccinated within a randomized trial of the recombinant zoster vaccine administered during or a maximum of 6 months after anti-lymphoma therapy, also showed low levels of seropositivity, varying between $20 \%$ and $50 \%{ }^{25}$

Currently available data point to the vital importance of COVID-19 prevention in cancer patients in general and in those with hematologic malignancies in particular. Evidence-based prophylactic approaches such as vaccination, have become the top priority measures significantly contributing to infection control. Nevertheless, the pivotal study, demonstrating $95 \%$ efficacy of the BNT162 b2 vaccine in COVID-19 prevention, did not include patients with lymphoma. In a single-center Israeli study, examining antibody-mediated response rates with the Elecsys anti-SARSCoV-2 $\mathrm{S}$ assay in patients with chronic lymphocytic leukemia, positive humoral responses were observed in $52 \%$ of patients, compared to $100 \%$ in an age- and sexmatched control cohort. ${ }^{26}$ Notably, the assay used in the latter study differed from the one employed in our analysis.

In the current study, treatment with anti-CD20 monoclonal antibodies as well as active disease at the time of vaccination emerged as significant predictors of a lack of serological response to BNT162b2. Likewise, the impact of anti-CD20 therapy was evident in the observed titers of anti-spike IgG antibodies, which increased as the time

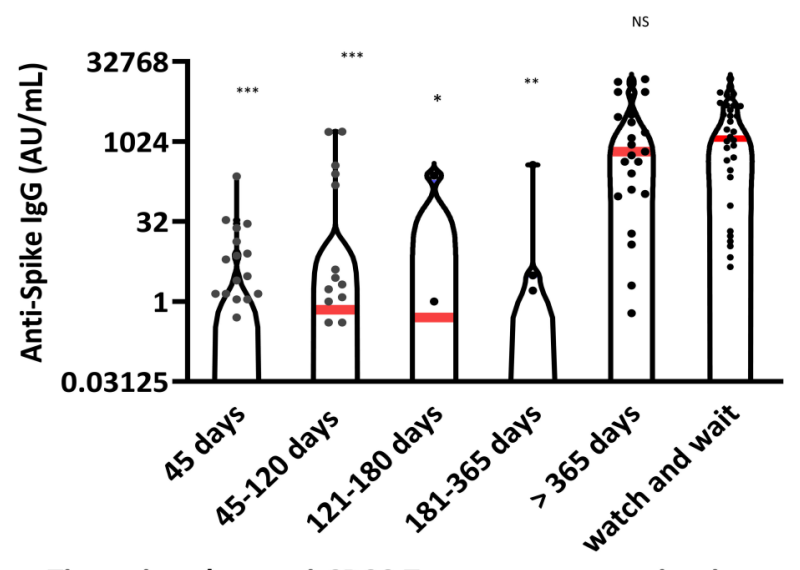

Time since last anti-CD20 Treatment to vaccination

Figure 1. Correlation between the levels of circulating anti-spike IgG antibodies and the time from the last anti-CD20 treatment to vaccination. Dots represent antibody titer values in arbitrary units (AU); red lines represent medians. $\star * * P<0.0001 ; * * P<0.001 ; * P<0.01$; NS: not statistically significant. 
period between the last anti-CD20 administration and vaccination became longer. With a cutoff of 12 months, our findings demonstrated a significant difference in the antibody titers between patients vaccinated less than or more than 12 months after anti-CD20 therapy, while the impact of exposure to this therapy became negligible after this time point. Actually, the titers became similar to those found in naïve (untreated) lymphoma patients. A plausible explanation could be that rituximab and other anti-CD20 monoclonal antibodies commonly used for the treatment of B-cell lymphoma lead to prolonged Bcell depletion and subsequent hypogammaglobulinemia. Consistent with our results, several studies reported data suggesting lower likelihoods of developing a serological response following anti-CD20 treatment in immunocompromised patients. For instance, patients with rheumatoid arthritis were reported to have lower titers of antiinfluenza antibodies upon treatment with rituximab compared to patients with rheumatoid arthritis not receiving such therapy. ${ }^{27}$ In another study, none of the 67 lymphoma patients vaccinated against influenza A (H1N1) within 6 months of receiving rituximab-containing regimens developed an antibody-mediated response compared to $82 \%$ in the control group. ${ }^{28}$ Finally, in the recently published study including a small cohort of antiCD20-treated chronic lymphocytic leukemia patients, none of those treated with rituximab within a year prior to vaccination developed anti-spike antibodies against SARS-CoV-2. ${ }^{26}$

Remarkably, in our study, patients receiving rituximab demonstrated an attenuated serological response to the vaccine, whereas patients treated with obinutuzumab failed to generate any anti-spike antibodies during the study period. This could be attributed to differences in pharmacodynamic properties between these two agents, as observed in in vitro studies, showing enhanced direct cell death and antibody-dependent cellular cytotoxicity for obinutuzumab compared to rituximab. ${ }^{29}$

In the present study, active disease emerged as an additional factor negatively affecting the humoral response to vaccine. While this could reflect the time-wise proximity to anti-CD20 treatment in these patients, it could also be associated with the effect of chemotherapeutic agents and corticosteroids commonly used during induction therapy in this clinical setting. Since humoral immunity requires functional $\mathrm{T}$ cells for the development of memory B cells and plasma cells, ${ }^{30,31}$ agents such as bendamustine and high-dose steroids, applied in lymphoma, might impede the serological response.

This study has several limitations, the lack of a control group being one of them. Nevertheless, Grupper et al., utilizing the same assay as in our study, showed that all healthy individuals included in the control group developed a serological response to the BNT162b2 vaccine. ${ }^{32}$ In addition, nucleocapsid antibody assessment was not part of the current analysis, since only patients with no documented febrile or respiratory events within months prior to vaccination were included in the study. Hence, the generation of anti-spike antibodies in response to subclinical COVID-19, while being possible, was unlikely in this population of patients.

A potential relationship between a weak serological response and the true protection from clinical COVID-19 will only become evident with longer follow-up. However, these data might never mature, as presently the pandemic has significantly subsided in Israel. Moreover, there are several newly validated assays capable of examining cellular immune responses to the vaccine, which will be included in future studies aimed at better understanding the true extent of protective immunity achieved with this vaccine.

In conclusion, the current study has shown that a heterogeneous group of lymphoma patients has developed attenuated serological responses to the BNT621b2 vaccine. Patients recently treated with anti-CD20 monoclonal antibodies ( $<12$ months since the last anti-CD20 treatment) are less likely to develop a serological response to this vaccine.

\section{Disclosures \\ No conflicts of interest to disclose.}

\section{Contributions}

$R G, S R-H$ and NAH designed and performed research, interpreted the data, and wrote the paper. UR designed research, analyzed the data, and wrote the paper. MS and HB-Z performed serology assays. GL, AG-G, CL, PR, MT-A, EJD, NH, TI, IT, $N L$ and RF collected data. All authors approved the final version of the paper.

\section{Aknowledgments}

We would like to thank Mrs. Sonia Kamenetsky for her advice and assistance in the preparation of this manuscript.

\section{Funding}

This research was supported by grants from Janssen, Takeda, Gilead Sciences and Abbvie. The Abbot test kits were kindly provided by the Israeli Ministry of Health.

\section{Data-sharing statement:}

The data that support the findings of this study are available from the corresponding author upon reasonable request.

\section{References}

1. WHO Coronavirus (COVID-19) Dashboard. Available at https://covid19.who.int/. Last accessed in June, 2021.

2. Azzi Y, Bartash R, Scalea J, Loarte-Campos P, Akalin E. COVID-19 and solid organ transplantation: a review article. Transplantation. 2021;105(1):37-55.

3. Lee LYW, Cazier JB, Starkey T, et al. COVID-19 prevalence and mortality in patients with cancer and the effect of primary tumour subtype and patient demographics: a prospective cohort study. Lancet Oncol. 2020;21(10):1309-1316.

4. Erdal GS, Polat O, Erdem GU, et al. The mortality rate of COVID-19 was high in cancer patients: a retrospective single-center study. Int J Clin Oncol. 2021;26(5):826834.

5. Rubinstein SM, Steinharter JA, Warner J, Rini BI, Peters S, Choueiri TK. The COVID19 and Cancer Consortium: a collaborative effort to understand the effects of COVID19 on patients with cancer. Cancer Cell. 2020;37(6):738-741.

6. Robilotti EV, Babady NE, Mead PA, et al. Determinants of COVID-19 disease severity in patients with cancer. Nat Med. 2020;26(8):1218-1223.

7. Vijenthira A, Gong IY, Fox TA, et al. Outcomes of patients with hematologic malignancies and COVID-19: a systematic review and meta-analysis of 3377 patients. Blood. 2020;136(25):2881-2892.

8. Baker D, Roberts CAK, Pryce G, et al. COVID-19 vaccine-readiness for antiCD20-depleting therapy in autoimmune diseases. Clin Exp Immunol. 2020;202(2): 149-161.

9. Morrison VA. Infections in patients with leukemia and lymphoma. Cancer Treat Res. 2014;161:319-349.

10. Dai M, Liu D, Liu M, et al. Patients with cancer appear more vulnerable to SARSCoV-2: a multicenter study during the COVID-19 outbreak. Cancer Discov. 2020;10(6):783-791. 
11. Shah V, Ko Ko T, Zuckerman M, et al. Poor outcome and prolonged persistence of SARS-CoV-2 RNA in COVID-19 patients with haematological malignancies; King's College Hospital experience. Br J Haematol. 2020;190(5):e279-e282.

12. Vabret N, Britton GJ, Gruber C, et al. Immunology of COVID-19: current state of the science. Immunity. 2020;52(6):910-941.

13. Dulery R, Lamure S, Delord $M$, et al. Prolonged in-hospital stay and higher mortality after Covid-19 among patients with non-Hodgkin lymphoma treated with Bcell depleting immunotherapy. Am J Hematol. 2021;96(8):934-944.

14. Polack FP, Thomas SJ, Kitchin N, et al. Safety and efficacy of the BNT162b2 mRNA Covid-19 vaccine. N Engl J Med. 2020;383(27):2603-2615.

15. Cheuk DK, Chiang AK, Lee TL, Chan GC, Ha SY. Vaccines for prophylaxis of viral infections in patients with hematological malignancies. Cochrane Database Syst Rev. 2011(3):CD006505.

16. Auletta J, Chemaly R, Khawaja F, et al. General principles of COVID-19 vaccines for immunocompromised patients. Available at https://www.hematology.org/ covid-19/ash-astct-covid-19-and-vaccines. Last accessed in April 2021

17. Interim clinical considerations for use of COVID-19 vaccines currently authorized in the United States. Available at https://www.cdc.gov/vaccines/covid19/info-by-product/clinical-considerations.html\#underlying-conditions. Last accessed in April 2021

18. He W, Chen L, Chen L, et al. COVID-19 in persons with haematological cancers. Leukemia. 2020;34(6):1637-1645

19. Pleyer C, Ali MA, Cohen JI, et al. Effect of Bruton tyrosine kinase inhibitor on efficacy of adjuvanted recombinant hepatitis $B$ and zoster vaccines. Blood. 2021;137(2):185-189.

20. Shah GL, DeWolf S, Lee YJ, et al. Favorable outcomes of COVID-19 in recipients of hematopoietic cell transplantation. J Clin Invest. 2020;130(12):6656-6667.

21. O'Nions J, Muir L, Zheng J, et al. SARSCoV-2 antibody responses in patients with acute leukaemia. Leukemia. 2021;35(1): 289-292.

22. Sun C, Pleyer C, Wiestner A. COVID-19 vaccines for patients with haematological conditions. Lancet Haematol. 2021;8(5): e312-e314.

23. Swerdlow SH, Campo E, Pileri SA, et al The 2016 revision of the World Health Organization classification of lymphoid neoplasms. Blood. 2016;127(20):2375-2390

24. Mazza JJ, Yale SH, Arrowood JR, et al. Efficacy of the influenza vaccine in patients with malignant lymphoma. Clin Med Res. 2005;3(4):214-220.

25. Dagnew AF, Ilhan O, Lee WS, et al Immunogenicity and safety of the adjuvanted recombinant zoster vaccine in adults with haematological malignancies: a phase 3 , randomised, clinical trial and posthoc efficacy analysis. Lancet Infect Dis. 2019;19(9):988-1000.

26. Herishanu Y, Avivi I, Aharon A, et al.
Efficacy of the BNT162b2 mRNA COVID19 vaccine in patients with chronic lymphocytic leukemia. Blood. 2021;137(23): 3165-3173.

27. Gelinck LB, Teng YK, Rimmelzwaan GF, van den Bemt BJ, Kroon FP, van Laar JM. Poor serological responses upon influenza vaccination in patients with rheumatoid arthritis treated with rituximab. Ann Rheum Dis. 2007;66(10):1402-1403.

28. Yri OE, Torfoss D, Hungnes $\mathrm{O}$, et al Rituximab blocks protective serologic response to influenza A (H1N1) 2009 vaccination in lymphoma patients during or within 6 months after treatment. Blood. 2011;118(26):6769-6771.

29. Tobinai K, Klein C, Oya N, FingerleRowson G. A review of obinutuzumab (GA101), a novel type II anti-CD20 monoclonal antibody, for the treatment of patients with B-cell malignancies. Adv Ther. 2017;34(2):324-356.

30. Haberman AM, Gonzalez DG, Wong P, Zhang TT, Kerfoot SM. Germinal center B cell initiation, GC maturation, and the coevolution of its stromal cell niches. Immunol Rev. 2019;288(1):10-27.

31. Nguyen DC, Joyner CJ, Sanz I, Lee FE. Factors affecting early antibody secreting cell maturation into long-lived plasma cells. Front Immunol. 2019;10:2138.

32. Grupper A, Sharon N, Finn T, et al. Humoral response to the Pfizer BNT162b2 vaccine in patients undergoing maintenance hemodialysis. Clin J Am Soc Nephrol. 2021;16(7):1037-1042. 